\title{
Numerical simulations of stripping effects in high-intensity hydrogen ion linacs
}

\author{
J.-P. Carneiro* \\ Fermi National Accelerator Laboratory, Batavia, Illinois 60510, USA \\ B. Mustapha and P. N. Ostroumov \\ Argonne National Laboratory, Argonne, Illinois 60439, USA
}

(Received 12 December 2008; published 27 April 2009; corrected 20 May 2009)

\begin{abstract}
Numerical simulations of $\mathrm{H}^{-}$stripping losses from blackbody radiation, electromagnetic fields, and residual gas have been implemented into the beam dynamics code TRACK. Estimates of the stripping losses along two high-intensity $\mathrm{H}^{-}$linacs are presented: the Spallation Neutron Source linac currently being operated at Oak Ridge National Laboratory and an $8 \mathrm{GeV}$ superconducting linac currently being designed at Fermi National Accelerator Laboratory.
\end{abstract}

DOI: 10.1103/PhysRevSTAB.12.040102

PACS numbers: 29.27.- a, 41.75.Cn

\section{INTRODUCTION}

Beam losses in an accelerator can be divided into two categories: controlled beam losses, achieved using a beam chopper, a collimator, or a beam dump, for example; and uncontrolled beam losses, arising from misalignment of accelerator components, rf phase and amplitude jitter in the accelerating cavities, beam halo formation, beam mismatch at rf frequency transitions, etc. In a high-power $\mathrm{H}^{-}$linac, another potential source of uncontrolled beam loss is the stripping of the $\mathrm{H}^{-}$ions. The $\mathrm{H}^{-}$ions have two electrons, one tightly bound with a binding energy of $13.6 \mathrm{eV}$ and another loosely bound with a binding energy of $0.75 \mathrm{eV}$. As the ions travel through the linac, they are subject to blackbody radiation, electromagnetic fields (Lorentz stripping), and collisions with residual gas molecules. Any one of these interactions may result in stripping of the loosely bound electron, which produces a neutral hydrogen atom. Because the neutral atom is no longer affected by the accelerating and focusing elements, it will generally produce uncontrolled beam loss. In this paper, we review the equations for $\mathrm{H}^{-}$stripping from blackbody radiation, electromagnetic fields, and residual gas. We describe the implementation of these equations into the beam dynamics code TRACK [1]. We present the results of numerical simulations of stripping losses along two high-intensity $\mathrm{H}^{-}$linacs: the Spallation Neutron Source (SNS) linac currently being operated at Oak Ridge National Laboratory and an $8 \mathrm{GeV}$ superconducting linac currently being designed at Fermi National Accelerator Laboratory.

The SNS linac is designed to deliver $1.6 \times 10^{14}$ protons on target with an rf pulse length of $1 \mathrm{msec}$ and an average current of $26 \mathrm{~mA}$ per pulse. At a kinetic energy of $1 \mathrm{GeV}$ and a repetition rate of $60 \mathrm{~Hz}$, the average beam power is

\footnotetext{
*Corresponding author. carneiro@fnal.gov
}

$\sim 1.4 \mathrm{MW}$. The FNAL Proton Driver is an $8 \mathrm{GeV}$ linac designed to deliver $1.56 \times 10^{14}$ protons to the main injector with a typical $\mathrm{rf}$ pulse length of $1 \mathrm{msec}$, producing an average beam current of $25 \mathrm{~mA}$ per pulse. At a kinetic energy of $8 \mathrm{GeV}$ and a repetition rate of $10 \mathrm{~Hz}$, the average beam power is $\sim 2 \mathrm{MW}$.

\section{STRIPPING LOSS ASSESSMENT}

One of the basic problems in the design and operation of high-power ion linacs is to keep the radioactivation of the beam line components low enough for "hands-on maintenance" which allows rapid access to the accelerator hardware and therefore results in higher machine availability. For hands-on maintenance with limited access time, activation levels must be below $100 \mathrm{mrem} / \mathrm{hr}$ at $30 \mathrm{~cm}$ from the component surface, after extended operation of the machine ( $\sim 100$ days) and four hours of down time [2]. Simulations and measurements for operating facilities such as the Los Alamos Neutron Science Center (LANSCE) $800 \mathrm{MeV}$ proton and $\mathrm{H}^{-}$linac and proton accumulator ring indicate this criterion corresponds to beam loss of about $1 \mathrm{~W} / \mathrm{m}$ or less [3]. This criterion is roughly independent of the beam energy for energies above $100 \mathrm{MeV}$ [4,5]. For energies below $100 \mathrm{MeV}$, the activation is less efficient, so that higher beam losses are acceptable.

Stripping losses of $0.1 \mathrm{~W} / \mathrm{m}$ correspond to a beam line activation of $10 \mathrm{mrem} / \mathrm{hr}$ at $30 \mathrm{~cm}$ four hours after shutdown. This allows unrestricted hands-on maintenance of the accelerator. Therefore beam losses from stripping below $0.1 \mathrm{~W} / \mathrm{m}$ are considered acceptable throughout this paper. Stripping losses between 0.1 and $1 \mathrm{~W} / \mathrm{m}$ are considered to be tolerable, but worthy of additional study to understand and minimize the sources of uncontrolled beam loss. We consider stripping losses above $1 \mathrm{~W} / \mathrm{m}$ to be unacceptable.

Figure 1 shows the permissible beam loss per unit length to achieve $0.1 \mathrm{~W} / \mathrm{m}$ as a function of the beam kinetic energy along the SNS linac and the FNAL $8 \mathrm{GeV}$ linac 


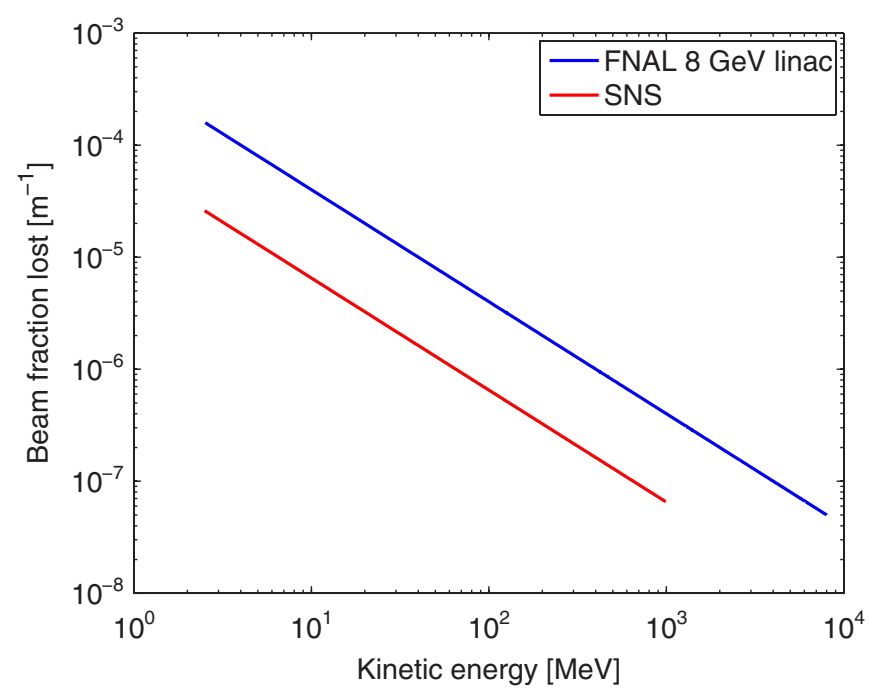

FIG. 1. (Color) Permissible beam loss fraction as a function of the beam kinetic energy to achieve $0.1 \mathrm{~W} / \mathrm{m}$ for the SNS linac operating at $1.4 \mathrm{MW}$ and the FNAL $8 \mathrm{GeV}$ linac operating at 2 MW.

operating at 1.4 and $2 \mathrm{MW}$, respectively. At the initial beam energy, stripping losses should not exceed $\sim 2.6 \times$ $10^{-5} \mathrm{~m}^{-1}$ for the SNS linac and $\sim 1.6 \times 10^{-4} \mathrm{~m}^{-1}$ for the FNAL $8 \mathrm{GeV}$ linac. As the energy increases, the acceptable beam loss fraction decreases. At the final beam energy, losses should not exceed $\sim 6.5 \times 10^{-8} \mathrm{~m}^{-1}$ for SNS and $\sim 5 \times 10^{-8} \mathrm{~m}^{-1}$ for the FNAL linac.

\section{REVIEW OF $\mathrm{H}^{-}$STRIPPING EFFECTS}

\section{A. Blackbody radiation stripping}

The spectral density $S$ of thermal photons per unit volume emitted by a beam pipe at a temperature $T$ is given by the Planck formula [6]

$$
S(\omega ; T) d \omega=\frac{\hbar}{\pi^{2} c^{3}} \frac{\omega^{3}}{\exp (\hbar \omega / k T)-1} d \omega,
$$

where $\omega$ is the photon angular frequency, $\hbar$ is the Planck constant divided by $2 \pi, c$ is the speed of light, and $k$ the Boltzmann constant. Integration of Eq. (1) for a beam pipe radiating at $300 \mathrm{~K}$ leads to approximately $5.47 \times 10^{14}$ photons per cubic meter. Figure 2 shows the photon spectral density for $T=300 \mathrm{~K}$ as a function of the photon energy in the laboratory frame and Doppler shifted into the rest frame of an $8 \mathrm{GeV}$ beam.

In the absence of an electric field, the photodetachment cross section of $\mathrm{H}^{-}$ions by thermal photons is given by [7]

$$
\sigma(E)=8 \sigma_{\max } \frac{E_{0}^{3 / 2}\left(E-E_{0}\right)^{3 / 2}}{E^{3}},
$$

where $E=\hbar \omega$ is the photon energy, $\sigma_{\max }=4.2 \times$ $10^{-21} \mathrm{~m}^{2}$ and $E_{0}=0.7543 \mathrm{eV}$, the lowest electron binding energy for $\mathrm{H}^{-}$. The photodetachment cross section as a

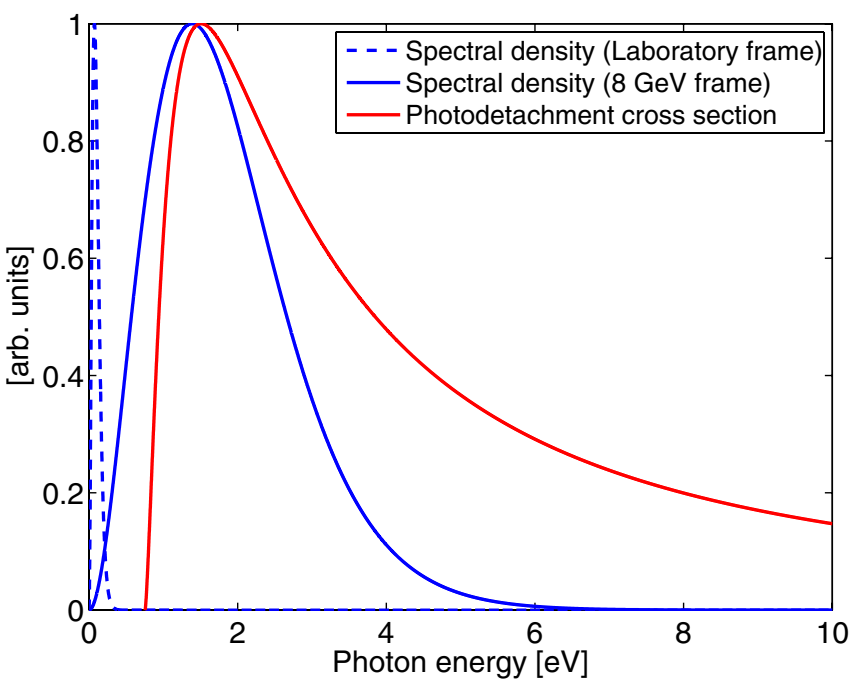

FIG. 2. (Color) Photon spectral density energy distribution at $T=300 \mathrm{~K}$ (in the laboratory frame and Doppler shifted to the beam frame) and $\mathrm{H}^{-}$photodetachment cross section.

function of photon energy is also shown in Fig. 2. The region of overlap between the photon spectral density distribution in the beam rest frame and the photodetachment cross section is where stripping of $\mathrm{H}^{-}$ions by blackbody radiation occurs. As can be seen in Fig. 2, the Doppler effect shifts the thermal photon energies upward and enhances the stripping probability dramatically.

Beam losses due to blackbody radiation stripping are presented by Bryant and Herling [8] and defined by the relation

$$
\frac{1}{L}=\int_{0}^{\infty} d \epsilon \int_{0}^{\pi} d \alpha \frac{d^{3} r}{d \epsilon d \alpha d l}
$$

where $\epsilon=E / E_{0}$ and $\alpha$ is the angle between the thermal photon and the $\mathrm{H}^{-}$beam. The double integral of Eq. (3) gives the beam fraction lost per unit length. The integrand of Eq. (3) can be expressed as

$$
\frac{d^{3} r}{d \epsilon d \alpha d l}=\frac{4 \pi E_{0}^{3} \epsilon^{2} \sin \alpha(1+\beta \cos \alpha) \sigma\left(\epsilon^{\prime}\right)}{(h c)^{3} \beta\left[\exp \left(\epsilon E_{0} / k T\right)-1\right]},
$$

where $\beta$ is the particle relative velocity and $\sigma\left(\epsilon^{\prime}\right)$ is the cross section in the beam rest frame. Integration of Eq. (3) for an $8 \mathrm{GeV}$ beam and a beam pipe at $300 \mathrm{~K}$ leads to a beam fraction lost of $\sim 7.86 \times 10^{-7} \mathrm{~m}^{-1}$, exceeding by 1 order of magnitude the acceptable stripping beam loss factor presented in Sec. II for the FNAL $8 \mathrm{GeV}$ linac operating at its full power. It is worth noting that this integral was done previously in Ref. [8] for the same beam kinetic energy and beam pipe radiation; their result was a bit smaller $\left(5.3 \times 10^{-7} \mathrm{~m}^{-1}\right)$. However, their result was obtained by integrating Eq. (3) approximately, so the two values are consistent when their respective accuracy is taken into account [9]. 
At cryogenic temperatures $(150 \mathrm{~K})$, Eq. (3) predicts a decrease of the beam fraction lost per meter to $\sim 2.51 \times$ $10^{-8} \mathrm{~m}^{-1}$ for an $8 \mathrm{GeV}$ beam, an acceptable level according to Sec. II. For a beam kinetic energy of $1 \mathrm{GeV}$ and a beam pipe temperature of $300 \mathrm{~K}$, the loss factor decreases to $\sim 2.7 \times 10^{-9} \mathrm{~m}^{-1}$, a tolerable level for the SNS linac operating at 1.4 MW, as discussed in Sec. II. Therefore, stripping losses due to blackbody radiation are of concern only for multi-GeV $\mathrm{H}^{-}$accelerators operating with beam pipes at room temperature and consequently are not expected to be a concern for the SNS linac.

In the presence of an external static electric field, Bryant et al. have observed a "ripple" structure in the photodetachment cross section [10]. This differs from the smooth photodetachment cross section shown in Fig. 2. It has been observed that the effect of the electric field is the largest when the polarization of the light is parallel to the electric field, while the effect is almost negligible for light polarized perpendicular to the electric field [11]. Du and Delos have interpreted the oscillatory behavior of the photodetachment cross section as arising from the interference between the electron wave going out from and returning to the nucleus following a closed orbit [12]. Recently, Du derived a formula for the photodetachment cross section in the presence of a static electric field $F$ which, for parallel polarization, takes the form [13]

$$
\sigma(E, F)=0.3604 \frac{F}{\left(E_{0}+E\right)^{3}} a_{0}^{2} D\left(\frac{2^{1 / 3} E}{F^{2 / 3}}\right)
$$

where $E$ and $F$ are in atomic units, $a_{0}$ is the Bohr radius of the hydrogen atom, and $D$ is a modulation factor which depends on the photon energy and the strength of the

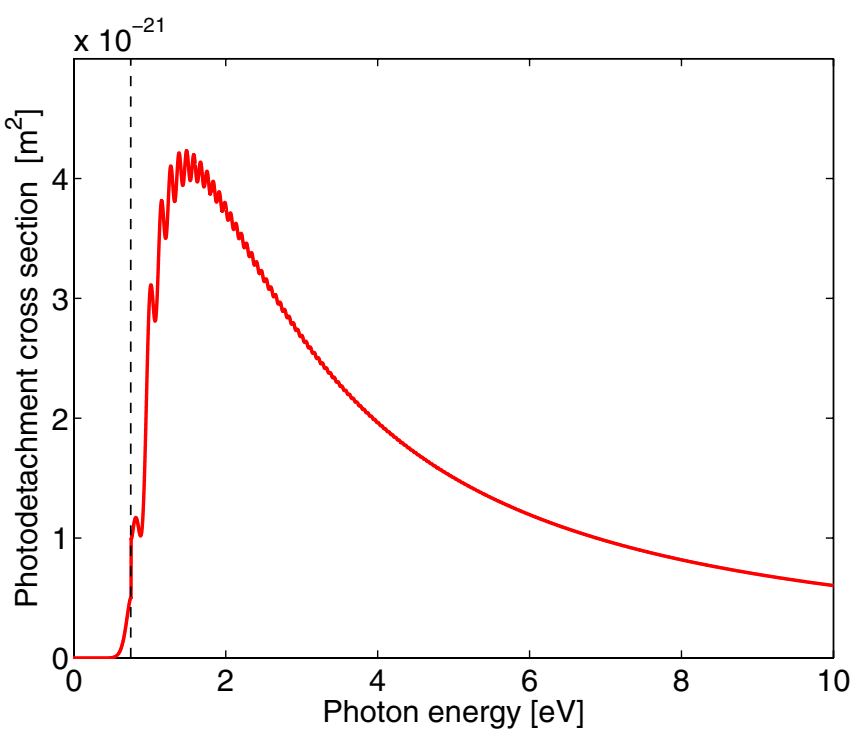

FIG. 3. (Color) $\mathrm{H}^{-}$photodetachment cross section in the presence of an external magnetic field $B=480 \mathrm{G}$. The dashed line indicates the threshold energy of $0.75 \mathrm{eV}$. electric field. Figure 3 shows the effect of an electric field of $136 \mathrm{MV} / \mathrm{m}$ on the photodetachment cross section for light polarized parallel to the electric field. This electric field amplitude corresponds to a magnetic field of $480 \mathrm{G}$ in the laboratory frame after a Lorentz transformation into the rest frame of an $8 \mathrm{GeV}$ beam. A magnetic field amplitude of $480 \mathrm{G}$ is typical of the field values foreseen in the dipoles for the high energy beam transfer line of the FNAL $8 \mathrm{GeV}$ linac, as will be discussed in Sec. V.

Interestingly, we see in Fig. 3 that, in the presence of an electric field, photodetachment occurs even below the zero-field energy threshold of $0.75 \mathrm{eV}$. In other words, stripping can occur even with a photon energy below the smallest electron binding energy of the $\mathrm{H}^{-}$ion. This can be interpreted as a quantum tunneling effect [12]. Above the threshold energy, the cross section oscillates about the zero-field cross section; the oscillation amplitude decreases as the photon energy increases. Consequently, we expect that the impact of the electric field on the cross section will not be significant when integrated over the photon energy. For the FNAL $8 \mathrm{GeV}$ linac with a transfer line at $300 \mathrm{~K}$, the electric field of $136 \mathrm{MV} / \mathrm{m}$ mentioned above increases the previously mentioned beam loss factor to $\sim 7.97 \times 10^{-7} \mathrm{~m}^{-1}$, a value consistent with the one presented in Ref. [8], which confirms the small contribution of the electric field to the photodetachment cross section.

The photodetachment cross section for photon energies up to $100 \mathrm{eV}$ has been computed by Broad and Reinhardt [14] and many experiments (most of them conducted at the LANSCE $800 \mathrm{MeV} \mathrm{H}^{-}$linac) have confirmed these observations. A summary of these experiments can be found in Ref. [15]. Doubly excited states in $\mathrm{H}^{-}$appear in the region from 10 to $15 \mathrm{eV}$, with two prominent resonances, the Feshbach resonance and the shape resonance, around $10.95 \mathrm{eV}$. For an $8 \mathrm{GeV}$ beam, because of the rapid decrease in the Doppler-shifted photon spectral density above $2 \mathrm{eV}$ or so, resonances in the photodetachment cross section at or above $10 \mathrm{eV}$ do not produce any significant change in the stripping probability. Hence, doubly excited states do not impact beam losses for the energy ranges considered herein, and the two-electron ejection cross section arising from blackbody radiation is not a concern for this paper.

\section{B. Lorentz stripping}

A strong electric field can remove one or even both electrons from an $\mathrm{H}^{-}$ion. Nevertheless, it is extremely difficult to achieve double electron detachment of a hydrogen atom in its ground state with a standard electric field [16], so Lorentz stripping is considered only for a single electron in this paper.

The rest-frame lifetime $\tau_{0}$ for single electron detachment of an $\mathrm{H}^{-}$ion subjected to an electric field is given by Scherk [17] and Keating [18] as 


$$
\tau_{0}=\frac{C_{1}}{F} \exp \left(\frac{C_{2}}{F}\right)
$$

where $F$ is the transverse electric field in the ion rest frame, $C_{1}=3.073 \times 10^{-6} \mathrm{~V} \mathrm{~s} / \mathrm{m}$ and $C_{2}=44.14 \times 10^{8} \mathrm{~V} / \mathrm{m}$. The values of $C_{1}$ and $C_{2}$ have been experimentally determined for an $800 \mathrm{MeV} \mathrm{H}^{-}$beam, and it is believed that they remain valid for energies up to $8 \mathrm{GeV}$ [19].

The beam fraction lost per unit length in the laboratory frame depends on the ion lifetime $\tau_{0}$,

$$
\frac{1}{L}=\frac{1}{\beta c \gamma \tau_{0}}
$$

where $\beta$ is the particle relative velocity, $c$ is the speed of light, and $\gamma$ is the Lorentz factor. Accelerating cavities and magnetic elements are possible sources for Lorentz stripping. Following the studies detailed in Ref. [20], Fig. 4 shows the transverse electric field at various radii for an $8 \mathrm{GeV} \mathrm{H}^{-}$beam in its own rest frame when it passes through a nine-cell ILC-type superconducting cavity. The cavity is operated in the accelerating mode $\left(\mathrm{TM}_{010} \pi\right)$ with an accelerating field on axis of $25 \mathrm{MV} / \mathrm{m}$ (a typical field foreseen for the FNAL $8 \mathrm{GeV}$ linac), with the phase set so that the beam is on crest. The largest possible transverse displacement is $34 \mathrm{~mm}$ (beyond which a particle will hit the cavity iris). A particle traveling with this maximum displacement experiences an average transverse electric field of order $F \simeq 112 \mathrm{MV} / \mathrm{m}$, which, from Eqs. (6) and (7), produces a negligible beam fraction lost of $\sim 1 \times$ $10^{-13} \mathrm{~m}^{-1}$. Therefore, we do not consider the stripping losses arising from the accelerating fields to be a concern for an $8 \mathrm{GeV} \mathrm{H}^{-}$linac.

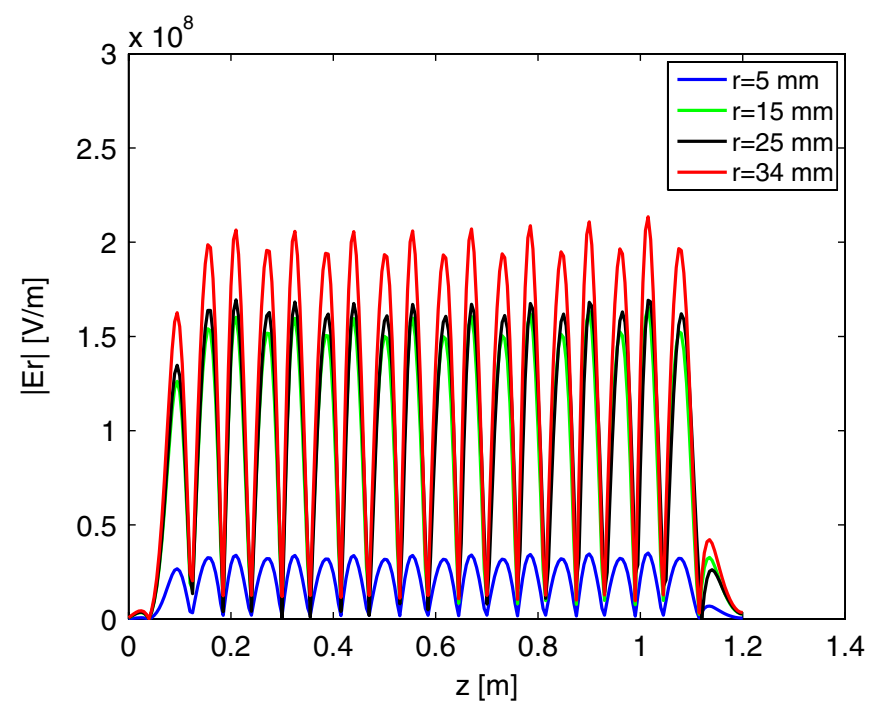

FIG. 4. (Color) Transverse electric field experienced by an $8 \mathrm{GeV}$ beam at various radii $r$ in an ILC cavity with an accelerating field of $25 \mathrm{MV} / \mathrm{m}$ with the beam on crest; $z$ is the axial coordinate along the cavity.
The same conclusion cannot be drawn concerning the stripping losses due to the magnetic fields. The electric field resulting from a Lorentz transformation of the magnetic field into the beam rest frame can become large enough to effectively strip $\mathrm{H}^{-}$ions in a multi-GeV linac. For this reason, the magnetic fields in transfer lines for $\mathrm{H}^{-}$ beams are kept to safe values to avoid stripping losses. For example, the SNS transfer line dipoles [21] have a magnetic field of $\sim 2088 \mathrm{G}$ for the $1 \mathrm{GeV}$ beam, corresponding to a beam-rest-frame electric field of $F \simeq 113 \mathrm{MV} / \mathrm{m}$ and a negligible beam fraction lost per unit length of $\sim 1.4 \times$ $10^{-13} \mathrm{~m}^{-1}$. For the dipoles of the FNAL $8 \mathrm{GeV}$ linac transfer line, the beam-rest-frame electric field is $F \simeq$ $136 \mathrm{MV} / \mathrm{m}$ (as discussed in Sec. III A), which corresponds to stripping losses of $\sim 1.25 \times 10^{-10} \mathrm{~m}^{-1}$. According to Sec. II, these stripping losses are negligible. Therefore, magnetic fields are not expected to play any significant role in stripping losses for the present configuration of the SNS and FNAL linacs.

\section{Residual gas stripping}

The $\mathrm{H}^{-}$beam can be stripped by interaction with the residual gas in the beam pipe. The lifetime $\tau_{m}$ of an $\mathrm{H}^{-}$ion in the presence of residual gas with a single molecular species $m$ is given by

$$
\tau_{m}=\frac{1}{d_{m} \sigma_{m} \beta c}
$$

where $d_{m}$ and $\sigma_{m}$ are the molecular density and interaction cross section for molecule $m$, respectively, and $\beta c$ is the speed of the $\mathrm{H}^{-}$beam. Assuming several molecular species in the residual gas, the total lifetime of the $\mathrm{H}^{-}$ion $\tau_{i}$ is given by

$$
\frac{1}{\tau_{i}}=\sum_{m} \frac{1}{\tau_{m}}
$$

The beam fraction lost per unit length is

$$
\frac{1}{L}=\frac{1}{\tau_{i} \beta c} .
$$

There is no data available above $800 \mathrm{MeV}$ for the electron-loss cross section of an $\mathrm{H}^{-}$ion, but a theoretical treatment is presented by Gillespie [22,23]. Gillespie uses the sum rule technique with the Born approximation to sum over all the final states of both the incident $\mathrm{H}^{-}$ion and the target atom of arbitrary atomic number $Z$ to calculate the total electron-loss cross section as a function of the energy of the incident $\mathrm{H}^{-}$ion. The total electron-loss cross section consists of two contributions: one arising from single electron detachment $\sigma_{-1,0}$ and one from double electron detachment $\sigma_{-1,1}$. The total electron-loss cross section can be expressed as [22]

$$
\begin{aligned}
\left(\sigma_{-1,0}+\sigma_{-1,1}\right)= & 8 \pi a_{0}^{2}\left(\frac{\alpha^{2}}{\beta^{2}}\right) \sum_{n \neq 0} \sum_{m}\left[I_{n m}-J_{n m}\left(\beta^{2}\right)\right. \\
& \left.-K_{n m}\left(\beta^{2}\right)\right]
\end{aligned}
$$


TABLE I. Total electron-loss cross section per molecule for $\mathrm{H}^{-}$in units of $10^{-18} \mathrm{~cm}^{2}$. Values for Xe were extracted from [23]; values for the other case were extracted from [24,25]. Values at $8 \mathrm{GeV}$ were obtained by scaling.

\begin{tabular}{lccccccccc}
\hline \hline Energy & $\mathrm{H}_{2}$ & $\mathrm{He}$ & $\mathrm{N}_{2}$ & $\mathrm{O}_{2}$ & $\mathrm{Ar}$ & $\mathrm{Xe}$ & $\mathrm{H}_{2} \mathrm{O}$ & $\mathrm{CO}_{2}$ & $\mathrm{CO}$ \\
\hline $5 \mathrm{keV}$ & $\ldots$ & $\ldots$ & $\ldots$ & $\ldots$ & $\ldots$ & $\ldots$ & 870 & $\ldots$ & 2000 \\
$1 \mathrm{MeV}$ & $\ldots$ & $\ldots$ & $\ldots$ & $\ldots$ & $\ldots$ & $\ldots$ & $\cdots$ & 620 & $\cdots$ \\
$10 \mathrm{MeV}$ & 2.1 & 6.04 & 30.7 & 32 & 83 & 500 & $\ldots$ & $\ldots$ & $\ldots$ \\
$8 \mathrm{GeV}$ & 0.04 & 0.12 & 0.65 & 0.68 & 1.75 & 10.59 & 0.01 & 1.33 & 0.02 \\
\hline \hline
\end{tabular}

where $a_{0}$ is the Bohr radius, $\alpha$ is the fine structure constant, $\beta$ is the particle relative speed, $n$ and $m$ are the final states of the $\mathrm{H}^{-}$ion and the target atom, respectively, $I_{n m}, J_{n m}$, and $K_{n m}$ integrals. Table I shows the total electron-loss cross section for $\mathrm{H}^{-}$ions interacting with various molecules. The values of the cross sections in the first three rows in Table I are based on measurements at lower energies and are taken as a reference to compute the cross sections at higher energies using the $1 / \beta^{2}$ scaling factor from Eq. (11). The last row in Table I reports cross sections scaled to $8 \mathrm{GeV}$.

References [23-25] present distinct measurements and theoretical predictions for the $\sigma_{-1,0}$ and $\sigma_{-1,1}$ cross sections for the above-mentioned atoms for energies up to $10 \mathrm{MeV}$. These results show that double electron detachment from a collision with an atom is not significant compared to single electron detachment: the ratio $\sigma_{-1,0} / \sigma_{-1,1}$ ranges from 20 for $\mathrm{H}_{2}$ to $>150$ for He.

The molecular density $d_{m}$ is related at $300 \mathrm{~K}$ to the pressure $P_{m}$ as [26]

$$
d_{m}\left[\mathrm{~m}^{-3}\right]=3.3 \times 10^{22} \cdot P_{m}[\text { torr }] .
$$

If the residual gas is composed of $100 \% \mathrm{H}_{2}$ at a pressure of $5 \times 10^{-6}$ torr, then Eq. (10) and Table I give a beam fraction lost per unit length at $8 \mathrm{GeV}$ of order $6.6 \times$ $10^{-7} \mathrm{~m}^{-1}$, which is unacceptable for the FNAL $8 \mathrm{GeV}$ linac operating at full power, according to Sec. II. A pressure of $5 \times 10^{-9}$ torr under the same conditions lowers the stripping losses by 3 orders of magnitude, to an acceptable level. Therefore, the residual gas pressure can have a significant impact on the stripping losses. Stripping due to residual gas is more pronounced at lower energies due to the larger electron-loss cross sections.

\section{IMPLEMENTATION IN THE BEAM DYNAMICS CODE TRACK}

The beam dynamics code TRACK [1] is the main tool for the design and simulation of the FNAL $8 \mathrm{GeV}$ linac. The code was developed by Argonne National Laboratory for beam dynamics modeling of proton and heavy ion linacs. The code integrates the equations of motion using a fourthorder Runge-Kutta method, with a variable integration step size defined for each element along the beam line. TRACK is
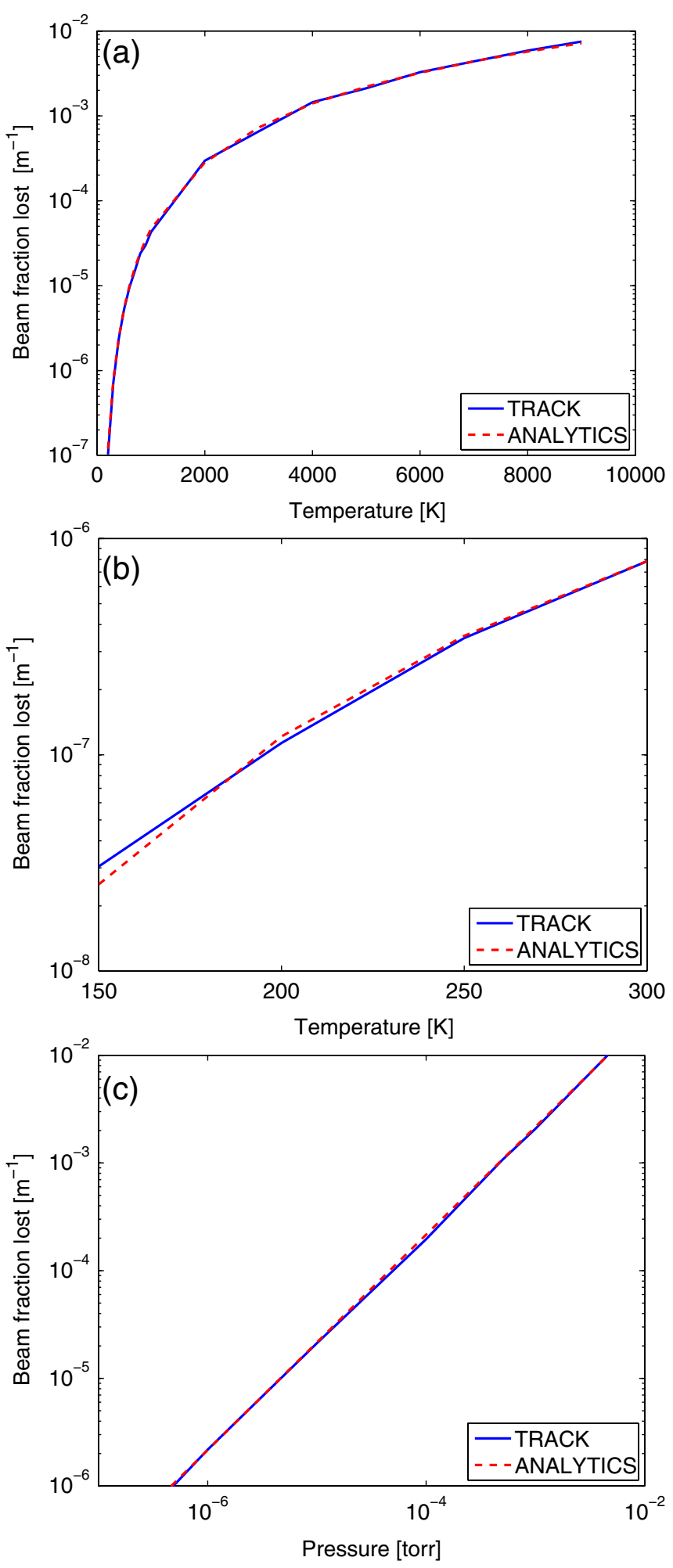

FIG. 5. (Color) Comparison between TRACK simulation and analytical prediction of the $\mathrm{H}^{-}$beam fraction lost at $8 \mathrm{GeV}$ from (a) blackbody radiation as a function of the beam pipe temperature; (b) Lorentz stripping as a function of the external magnetic field; and (c) residual gas stripping as a function of the beam pipe pressure. Residual gas with airlike composition is assumed. 
a fully 3D code: it uses 3D external electric and magnetic fields defined on a rectangular mesh and 3D space charge fields. The charge distribution is defined on a rectangular mesh using the "clouds-in-cell" method; the space charge fields are calculated in the beam rest frame via the Poisson equation. The boundary conditions used in TRACK are parallel conducting walls in the transverse dimensions and periodic boundary conditions in the longitudinal dimension.

Stripping losses from blackbody radiation are implemented in TRACK through Eq. (2), i.e., neglecting the effect of the electric field on the photodetachment cross section, as discussed in Sec. III A. For the losses from electromagnetic fields and residual gas, Eqs. (7) and (10) are used in the code, respectively. TRACK allows the user to set the temperature, pressure, and composition of the residual gas for any desired section of the linac. The code allows for stripping from the molecules listed in Table I. At each integration step, the code calculates a stripping probability for every macroparticle and compares this probability with a uniformly generated random number between 0 and 1 . In the event the random number is smaller than the calculated probability, the particle will be stripped. In this paper, stripped particles are considered lost at the location of stripping without further tracking.

Figure 5 compares TRACK simulations of the stripping losses from the three mechanisms with analytical predictions. The simulations are for an $8 \mathrm{GeV} \mathrm{H}^{-}$beam going through residual gas with airlike composition. The overall agreement is excellent and confirms the proper implementation of the stripping equations in TRACK.

\section{APPLICATION TO HIGH-INTENSITY HYDROGEN ION LINACS}

Losses from stripping effects were studied with the code TRACK for two high-intensity hydrogen ion linacs: the Spallation Neutron Source (SNS), in operation since June 2006 at Oak Ridge National Laboratory (ORNL), and an $8 \mathrm{GeV}$ superconducting linac currently under development at Fermi National Accelerator Laboratory. Baseline parameters for these two accelerators are given in Table II.

\section{A. The Spallation Neutron Source}

The Spallation Neutron Source [27] is a short-pulse neutron source designed to deliver a $1 \mathrm{GeV}$ proton beam with $1.4 \mathrm{MW}$ average power onto a liquid mercury target for neutron spallation. The accelerator complex consists of an $\mathrm{H}^{-}$injector, a linear accelerator, and a high energy beam transfer line (HEBT) that connects the linac to the injection point in the accumulator ring. The beam is accumulated over 1060 turns before extraction for delivery to the liquid mercury target. A layout of the SNS linac and HEBT is shown in Fig. 6.
TABLE II. Baseline design parameters for the ORNL Spallation Neutron Source (ORNL SNS) and the FNAL Proton Driver (FNAL PD).

\begin{tabular}{lcc}
\hline \hline Parameter & ORNL SNS & FNAL PD \\
\hline Particle type & $\mathrm{H}^{-}$ & $\mathrm{H}^{-}$ \\
Beam energy & $1 \mathrm{GeV}$ & $8 \mathrm{GeV}$ \\
Beam average power & $1.4 \mathrm{MW}$ & $2 \mathrm{MW}$ \\
Beam pulse length & $1 \mathrm{msec}$ & $1 \mathrm{msec}$ \\
Pulse repetition rate & $60 \mathrm{~Hz}$ & $10 \mathrm{~Hz}$ \\
$\mathrm{H}^{-}$peak current from RFQ & $38 \mathrm{~mA}$ & $43.25 \mathrm{~mA}$ \\
Average current per macropulse & $26 \mathrm{~mA}$ & $25 \mathrm{~mA}$ \\
Particles per macropulse & $1.6 \times 10^{14}$ & $1.56 \times 10^{14}$ \\
Linac length & $\sim 335 \mathrm{~m}$ & $\sim 678 \mathrm{~m}$ \\
Transport line length & $\sim 172 \mathrm{~m}$ & $\sim 1 \mathrm{~km}$ \\
\hline \hline
\end{tabular}

The injector consists of an $\mathrm{H}^{-}$ion source followed by a radio-frequency quadrupole (RFQ) and a medium energy beam transport line (MEBT) that matches the $2.5 \mathrm{MeV}$ beam into the linear accelerator. The linac includes both room temperature and superconducting structures: a sixtank drift tube linac (DTL) accelerates the beam up to $87 \mathrm{MeV}$; a four-module coupled-cavity linac (CCL) then accelerates to $186 \mathrm{MeV}$; a superconducting linac (SCL) with $\beta=0.61$ cavities accelerates the beam up to $387 \mathrm{MeV}$; final acceleration to $1 \mathrm{GeV}$ in the SCL is provided by $\beta=0.81$ cavities. The front end of the linac (up to the DTL) operates at a frequency of $402.5 \mathrm{MHz}$; the rest of the linac operates at $805 \mathrm{MHz}$. The HEBT, consisting of eight bending magnets of $11.25^{\circ}$ each, prepares the beam by cleaning up the transverse and longitudinal halo during the transport to the accumulator ring injection point.

The layout of the SNS linac and HEBT were input into TRACK and stripping simulations were performed from the RFQ exit to the stripping foil at the accumulator ring injection point. The predicted beam losses from residual gas and blackbody radiation are shown in Fig. 7. For improved statistics, 100 simulations were run in parallel on FermiGrid [28] with a different seed for the random number generator in each case. The simulations were performed with $10^{6}$ macroparticles and the losses were scaled to a beam power of $1.4 \mathrm{MW}$. The temperature along the linac and HEBT was set to $300 \mathrm{~K}$ in the warm sections and $2.1 \mathrm{~K}$ in the cryomodules. For the residual gas pressure, typical values measured along the SNS linac [29] were used, ranging from $\sim 2.5 \times 10^{-7}$ torr in the MEBT to $\sim 1 \times 10^{-10}$ torr in the cryomodules and $\sim 2.5 \times$ $10^{-9}$ torr in the HEBT. A generic vacuum composition $\left(70 \% \mathrm{H}_{2}, 10 \% \mathrm{H}_{2} \mathrm{O}, 10 \% \mathrm{CO}_{2}\right.$, and $10 \% \mathrm{CO}$ ) was assumed in the warm section; $100 \% \mathrm{H}_{2}$ was assumed in the cryomodules. Figure 7(a) shows that, for the pressures considered in the simulations, the stripping losses from the residual gas remain below $0.1 \mathrm{~W} / \mathrm{m}$. The losses from residual gas are higher in the linac front end because the cross sections are larger at lower energy, as discussed in 


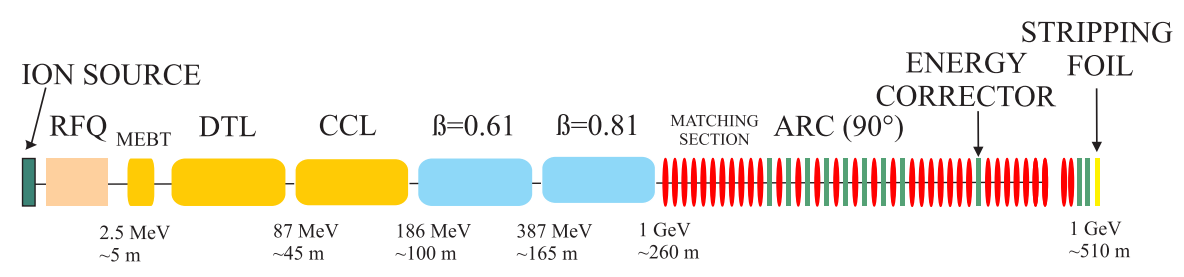

FIG. 6. (Color) Layout of the SNS linac and HEBT.

Sec. III C. As indicated in Fig. 7(b), TRACK predicts very limited losses from blackbody radiation $(\sim 2 \times$ $10^{-2} \mathrm{~W} / \mathrm{m}$ ), as expected from Sec. III A. These losses are located in the HEBT. The stripping losses from the electromagnetic fields were also computed with TRACK along the SNS linac and HEBT. The results show negligible losses $\left(<10^{-2} \mathrm{~W} / \mathrm{m}\right)$, as expected from Sec. III B. From these studies, we conclude that $\mathrm{H}^{-}$stripping losses from residual gas, blackbody radiation, and electromag-
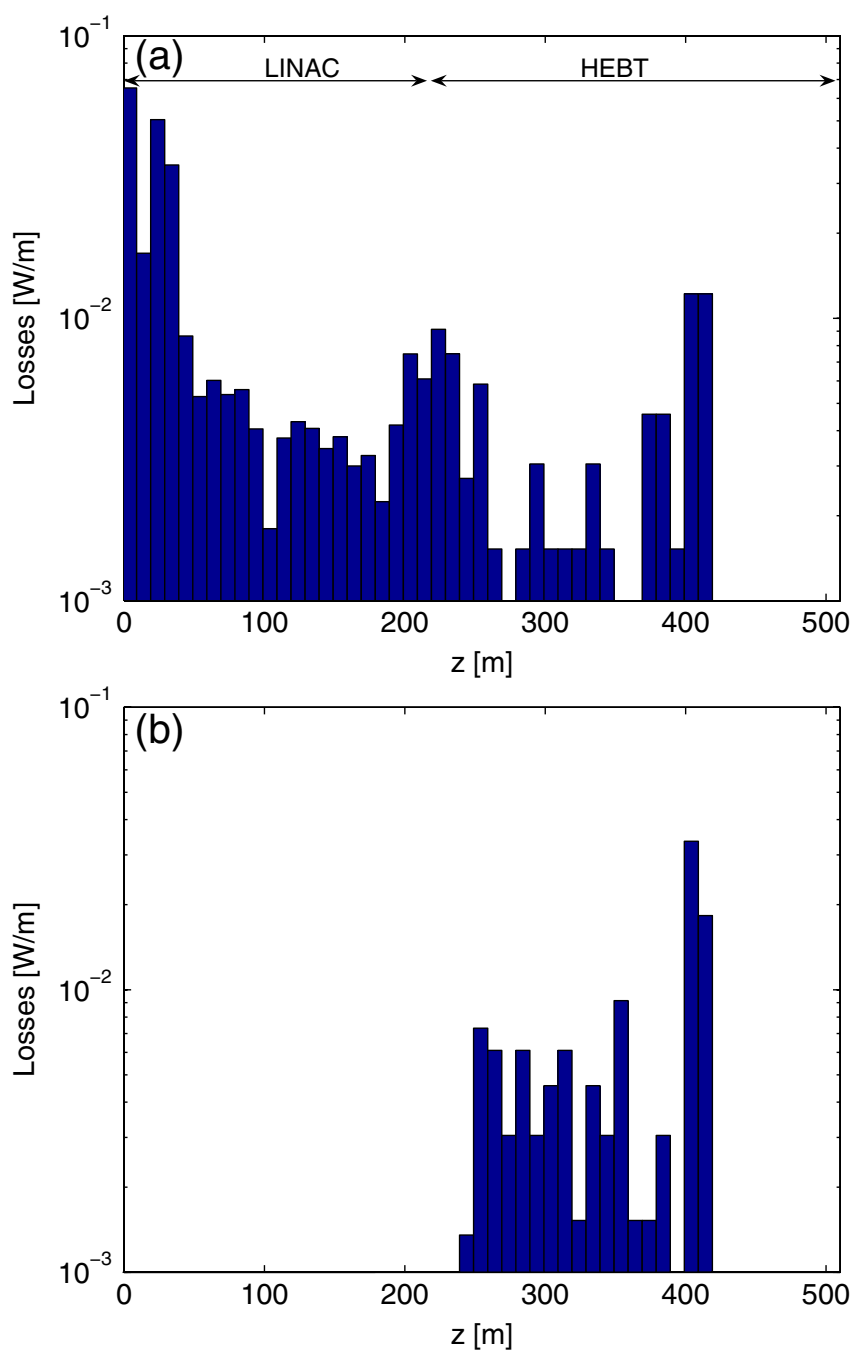

FIG. 7. (Color) TRACK simulation of the beam power lost per meter from (a) residual gas stripping and (b) blackbody radiation stripping along the SNS linac and HEBT with 1.4 MW beam power. netic fields remain below $0.1 \mathrm{~W} / \mathrm{m}$ along the SNS linac and HEBT for the residual gas pressures considered in this paper and therefore are acceptable, according to the requirements of Sec. II.

\section{B. The FNAL $8 \mathrm{GeV}$ superconducting linac}

The FNAL Proton Driver [30] is an $8 \mathrm{GeV} \mathrm{H}^{-}$superconducting linac conceived with the primary mission of providing a $2 \mathrm{MW}$ proton beam at main injector energies $(40-120 \mathrm{GeV})$ for the FNAL neutrino program. A layout of the linac and the high energy beam transfer line to the main injector is shown in Fig. 8.

Like SNS, the injector consists of an $\mathrm{H}^{-}$ion source and a $2.5 \mathrm{MeV}$ radio-frequency quadrupole (RFQ). In the linac front end, it was proposed to use superconducting (SC) spoke resonators [31] in lieu of the usual DTL and CCL structures. In fact, several SC spoke resonators can be powered by a single klystron with the use of fast ferrite phase shifters [32], in contrast to the usual power distribution system for normal conducting cavities in which each DTL tank and CCL structure is powered by a single klystron. As shown in Fig. 8, a MEBT section matches the beam into 16 room-temperature cross-bar H-type $(\mathrm{CH})$ cavities. Above $10 \mathrm{MeV}$, two types of single spoke resonators (SSR 1, SSR 2) and one type of triple spoke resonator (TSR) are used to accelerate the beam up to $\sim 420 \mathrm{MeV}$. At this energy, the frequency transition from $325 \mathrm{MHz}$ to $1.3 \mathrm{GHz}$ takes place, and the beam is further accelerated up to $8 \mathrm{GeV}$ using squeezed-ILC (S-ILC, $\left.\beta_{G}=0.81\right)$ and ILC $\left(\beta_{G}=1.0\right)$ cavities. Superconducting solenoids were selected as focusing elements in the linac front end. Above $\sim 120 \mathrm{MeV}$, focusing is done via focusing and defocusing quadrupole (FODO) channels, since focusing with $\sim 6 \mathrm{~T}$ solenoids can result in stripping of the $\mathrm{H}^{-}$beam. The ILC section of the linac is made of two types of cryomodules: the first type (ILC 1) contains two quadrupoles and seven ILC cavities; the second type (ILC 2) contains one quadrupole and eight cavities. As indicated in Fig. 8, the HEBT transports the beam from the linac to the main injector for multiturn charge-exchange injection through a stripping foil. The $\sim 1 \mathrm{~km}$ transfer line is made of two arcs of opposite bend directions, followed by four debuncher cavities to provide the necessary transverse and longitudinal beam matching to the main injector. Each arc contains 36 bending magnets of $0.56^{\circ}$ each.

Figures 9 and 10 show TRACK simulations along the FNAL $8 \mathrm{GeV}$ linac and HEBT of stripping losses due to 


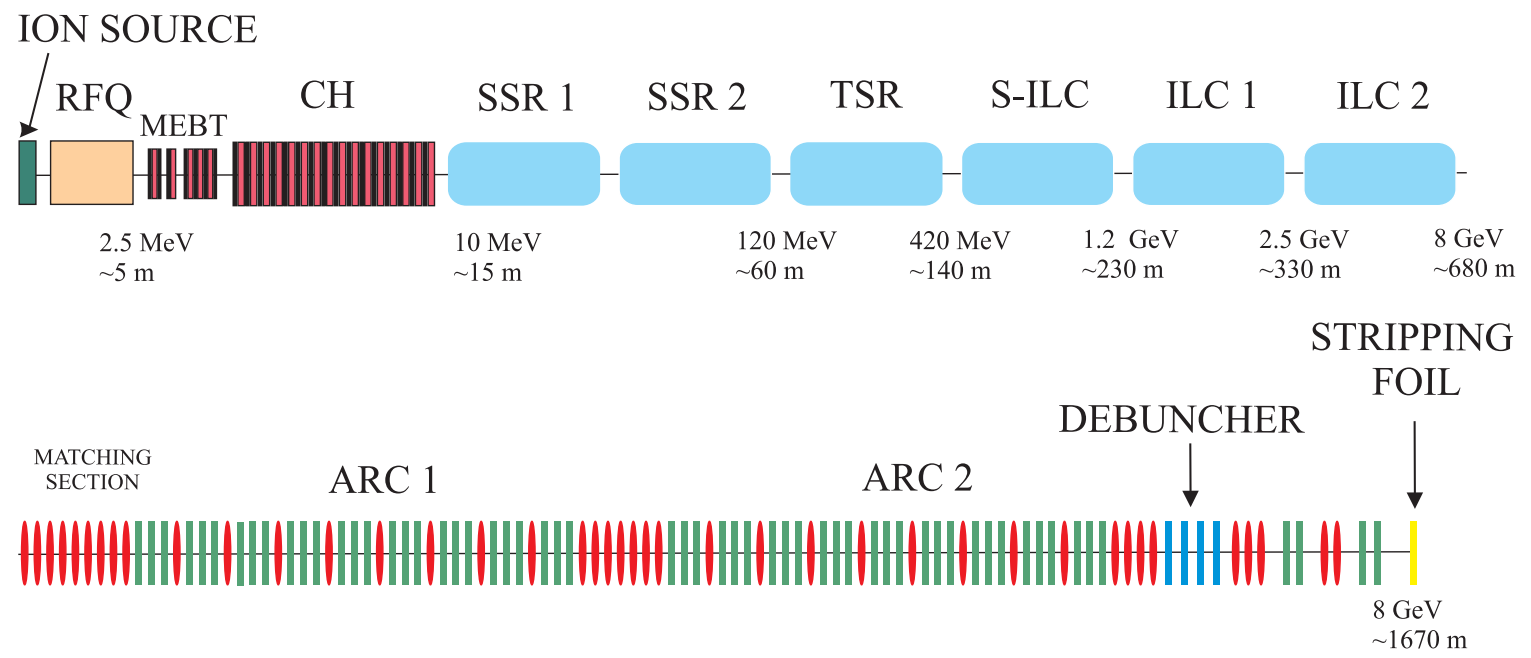

FIG. 8. (Color) Layout of the FNAL $8 \mathrm{GeV}$ linac and HEBT.
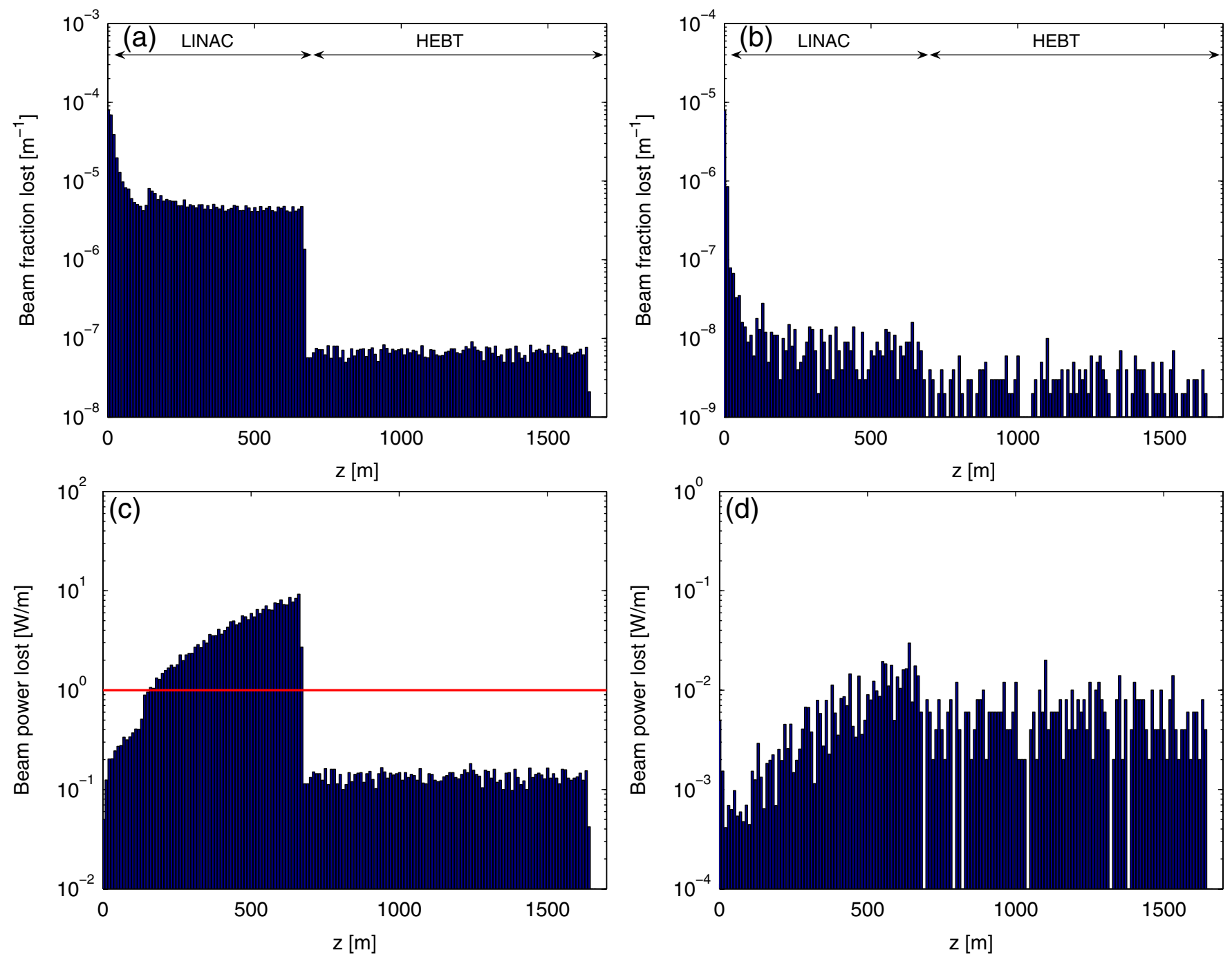

FIG. 9. (Color) TRACK simulations of the beam fraction lost per meter from residual gas stripping along the FNAL 8 GeV linac and HEBT at 2 MW beam power for (a) severe and (b) typical conditions; corresponding power loss per unit length for (c) severe and (d) typical conditions. The red line in (c) indicates the $1 \mathrm{~W} / \mathrm{m}$ limit. 
residual gas and blackbody radiation, respectively. Two operating conditions were studied: "severe" conditions intended to artificially generate high stripping losses, shown on the left in Figs. 9 and 10; and "typical" conditions anticipated for actual operation of the linac, shown on the right in Figs. 9 and 10. Under the severe conditions the pressure is $1 \times 10^{-7}$ torr along the linac and HEBT and the temperature of the HEBT is $300 \mathrm{~K}$. Under the typical conditions, pressure values typical of the measured values for SNS are assumed (i.e. $1 \times 10^{-7}$ torr in the roomtemperature sections, $1 \times 10^{-10}$ torr in the cryomodules, and $5 \times 10^{-9}$ torr in the HEBT, as discussed in the previous section) and the temperature of the transfer line is set to $150 \mathrm{~K}$. In all cases, the temperature of the SC solenoids and the SSR and TSR cryomodules was set to $4 \mathrm{~K}$ and the temperature of the S-ILC and ILC cryomodules was $2 \mathrm{~K}$. Warm sections were assumed between cryomodules. As in the SNS simulations, a residual gas made of $70 \% \mathrm{H}_{2}, 10 \%$
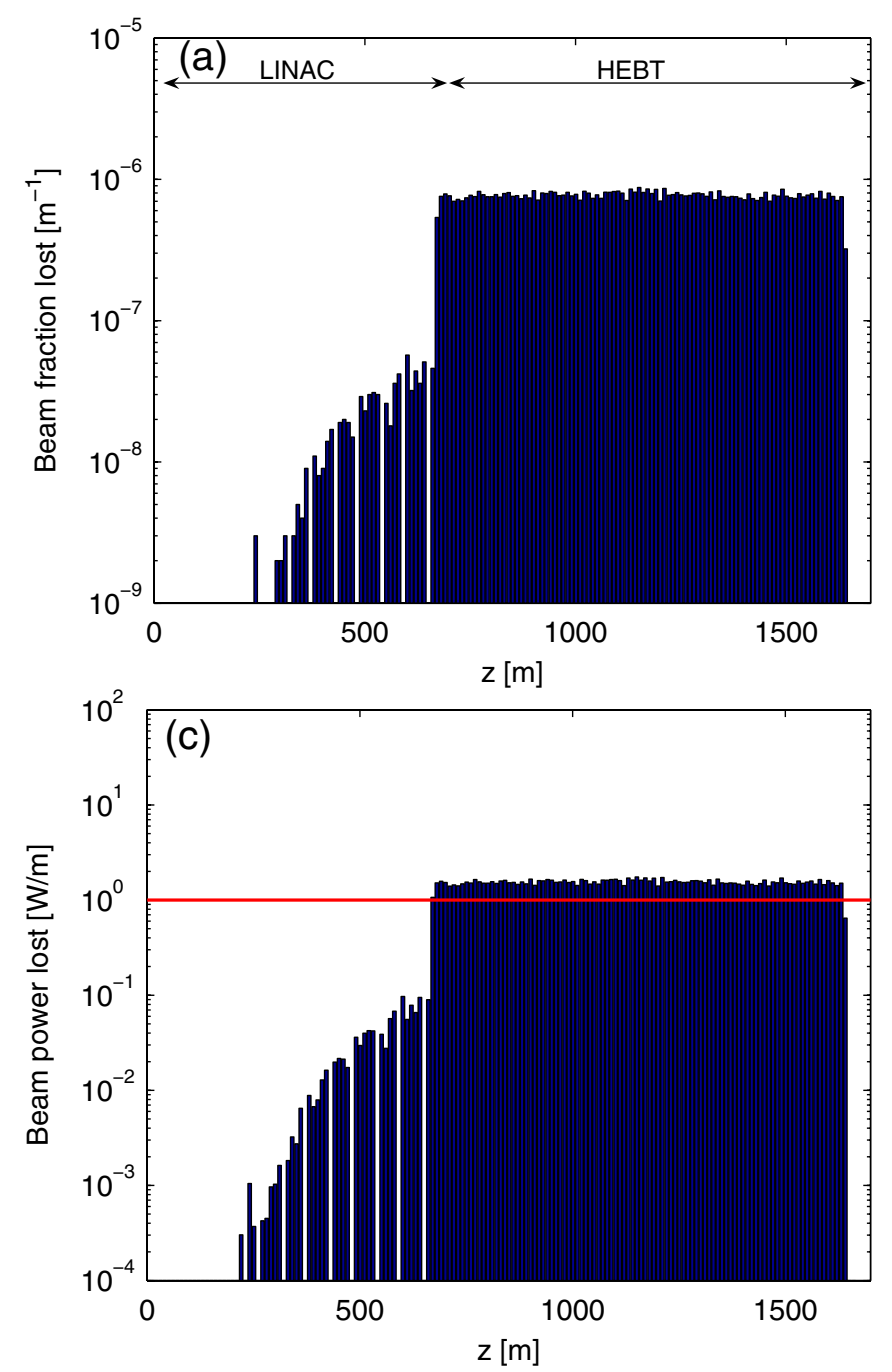

$\mathrm{H}_{2} \mathrm{O}, 10 \% \mathrm{CO}_{2}$, and $10 \% \mathrm{CO}$ was assumed for the warm sections and $100 \% \mathrm{H}_{2}$ was assumed for the cryomodules. The simulations were performed on FermiGrid in the same configuration as the SNS stripping simulations (statistics on $10^{8}$ macroparticles) and the results were scaled to $2 \mathrm{MW}$ beam power.

The effect of the residual gas pressure on the stripping losses can be seen clearly in Fig. 9: the poor vacuum [Fig. 9(a)] produces a beam fraction lost along the linac and HEBT ranging from $\sim 10^{-4}$ to $\sim 7 \times 10^{-8} \mathrm{~m}^{-1}$ while the typical vacuum [Fig. 9(b)] reduces the beam fraction lost by 1 order of magnitude or more. Figure 9(a) shows clearly the impact of the beam energy in the cross section between the hydrogen ion and the residual gas molecules: at low energy, the cross section is larger, inducing a larger beam fraction loss; at high energy, the cross section decreases, as expected from Eq. (11), leading to lower beam fraction lost. It is interesting to notice in Fig. 9(c) that, in
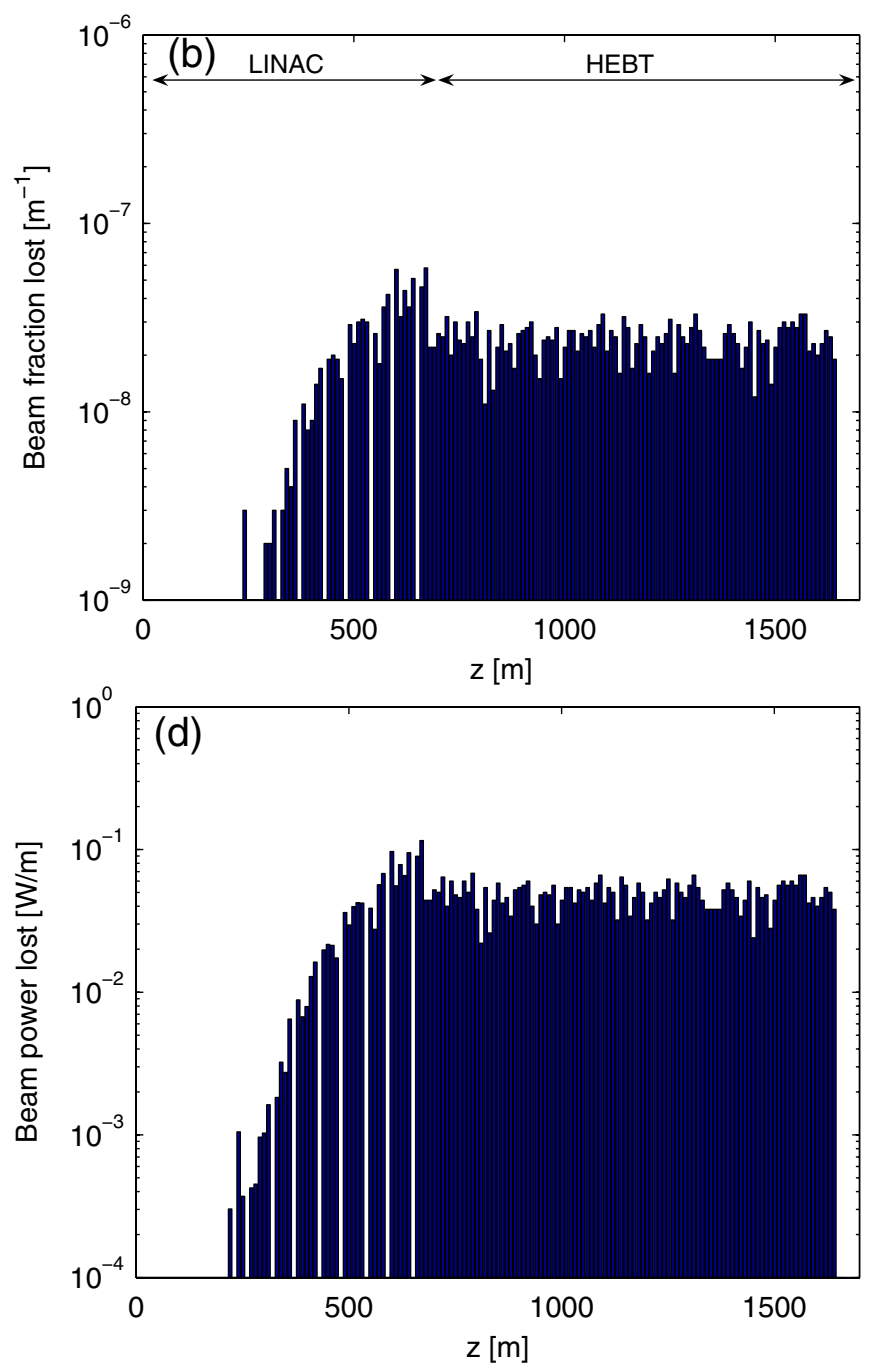

FIG. 10. (Color) TRACK simulations of the beam fraction lost per meter from blackbody radiation stripping along the FNAL 8 GeV linac and HEBT at 2 MW beam power for (a) severe and (b) typical conditions; corresponding power loss per unit length for (c) severe and (d) typical conditions. The red line in (c) indicates the $1 \mathrm{~W} / \mathrm{m}$ limit. 
spite of the decreasing beam fraction lost per meter in the linac, the power loss per meter is increasing, due to the increasing beam energy. A rapid decrease in the beam fraction lost per meter (from $\sim 4 \times 10^{-6}$ to $\sim 7 \times$ $10^{-8} \mathrm{~m}^{-1}$ ) is observed in Fig. 9(a) when going from the SC linac to the HEBT. This rapid decrease in losses is due to the decrease in the molecular density of the residual gas which accompanies the increase in temperature at constant pressure (going from gas at $2.1 \mathrm{~K}$ in the linac section to gas at $300 \mathrm{~K}$ in the HEBT, while the pressure remains $1 \times$ $10^{-7}$ torr). We conclude from these studies that, for typical pressures, the stripping losses due to residual gas remain below $\sim 2 \times 10^{-2} \mathrm{~W} / \mathrm{m}$ [Fig. 9(d)], an acceptable level as discussed in Sec. II.

The impact of the temperature on losses due to blackbody radiation can be seen in Fig. 10. With the HEBT at $300 \mathrm{~K}$ [Fig. 10(a)], the beam fraction lost reaches $\sim 7 \times$ $10^{-7} \mathrm{~m}^{-1}$; cooling the HEBT to $150 \mathrm{~K}$ [Fig. 10(b)] decreases the beam fraction lost to $\sim 2 \times 10^{-8} \mathrm{~m}^{-1}$. At $300 \mathrm{~K}$ the power loss per meter along the HEBT [Fig. 10(c)] exceeds the $1 \mathrm{~W} / \mathrm{m}$ limit criterion of Sec. II. At $150 \mathrm{~K}$ [Fig. 10(d)] the losses remain below $\sim 5 \times$ $10^{-2} \mathrm{~W} / \mathrm{m}$ and are acceptable. It is interesting to note in Figs. 10(a) and 10(b) that some stripping losses are observed in the linac section. These losses take place between the cryomodules, where the beam line was considered to be warm. The corresponding beam power lost [Figs. 10(c) and 10(d)] remains below the acceptable limit of $0.1 \mathrm{~W} / \mathrm{m}$. From these studies, we conclude that cooling the HEBT to $150 \mathrm{~K}$ or lower will be crucial for operation of the FNAL $8 \mathrm{GeV}$ linac at $2 \mathrm{MW}$ beam power. Furthermore, operating the HEBT at cryogenic temperatures will improve the vacuum and hence reduce stripping losses from the residual gas.

We conclude from the simulations presented in Figs. 9 and 10 that, for typical pressures and with operation of the HEBT at $150 \mathrm{~K}$ or lower, the stripping losses do not exceed the acceptable limit of $0.1 \mathrm{~W} / \mathrm{m}$ from Sec. II. Moreover, as discussed in Sec. III B, stripping from the present magnetic field configuration is not of concern for the FNAL $8 \mathrm{GeV}$ linac and HEBT. This was checked with additional simulations, which indicated that negligible stripping losses $\left(<1 \times 10^{-3} \mathrm{~W} / \mathrm{m}\right)$ occur in the HEBT due to the magnetic field.

\section{DISCUSSION AND FURTHER DEVELOPMENTS}

The simulations presented in Secs. VA and V B provide two examples to show that stripping losses due to blackbody radiation, electromagnetic fields, and residual gas can be modeled with the code TRACK. To accurately simulate these three effects, the temperature of the beam pipe, the electromagnetic fields, and the pressure profile along the linac need to be accurately input to the code.
Particular attention is needed with the residual gas pressure, since several mechanisms can affect it. For instance, in Secs. VA and VB the residual gas pressure along the major parts of the SNS and FNAL linacs was assumed constant. In reality, however, the residual gas pressure will generally vary as a function of position. In the case of a room-temperature linac or transport line, the residual gas pressure usually has maxima between the pressure gauges which are commonly located near the vacuum pumps. As a result, the maximum residual gas pressure could be as much as 1 order of magnitude higher than the measured values [33]. The opposite is likely to be true in the case of a linac or transport line at cryogenic temperature. In fact, in the cryogenic case, the pressure gauges and the vacuum pumps are typically at room temperature (between cryomodules, for example) but the cold surfaces are able to pump much more efficiently (by cryopumping). As a consequence, we expect the pressure to be smaller than the value indicated by the gauges in the cryogenic case.

In light of these considerations, the stripping losses presented in Figs. 9(b) and 9(d) for the FNAL $8 \mathrm{GeV}$ linac operating with an HEBT at $150 \mathrm{~K}$ are likely to be an upper limit scenario, since the beam line is at cryogenic temperatures nearly everywhere. In the SNS case, where significant portions of the beam line are warm, we repeated the TRACK simulations of Fig. 7(a) with more severe residual gas pressure. We considered a residual gas pressure higher by 1 order of magnitude in the room-temperature sections of the linac (MEBT, DTL, CCL, HEBT, and spaces between cryomodules). In these simulations, we observed, according to the stripping loss assessment defined in Sec. II, tolerable losses (up to $\sim 0.7 \mathrm{~W} / \mathrm{m}$ ) in the MEBT and DTL sections and acceptable losses $\left(<10^{-2} \mathrm{~W} / \mathrm{m}\right)$ from the CCL section to the end of the linac. The stripping losses in the MEBT and DTL sections occur at an energy below $100 \mathrm{MeV}$ and therefore are expected to activate the beam line less efficiently, as discussed in Sec. II. These simulations confirm that, for the configuration of the residual gas pressure presented in Sec. VA along the SNS linac, we do not expect the stripping losses from the residual gas to be of a concern.

It is also important to point out that beam losses in high vacuum systems can degrade the residual gas pressure via ion-stimulated gas desorption, scattering (Coulomb or Bremsstrahlung), or charge-exchange, for example [34]. These mechanisms are particularly important in the lowpressure sections of the linac $\left(<10^{-9}\right.$ torr). The evolution of the residual gas pressure along an accelerator can be modeled with codes like VAKTRAK [35], MOLFLOW [36], or STRAHLSIM [37]. These codes calculate the pressure profiles for a given array of pumps, outgassing elements, and beam pipes. Interfacing TRACK to the output from one of these codes should allow for better predictions of stripping losses from residual gas in the future. 


\section{CONCLUSION}

Simulations of stripping losses from blackbody radiation, electromagnetic fields (Lorentz stripping), and residual gas interactions are now available in the beam dynamics code TRACK. Numerical simulations of the SNS linac and transport line have shown acceptable stripping losses $(<0.1 \mathrm{~W} / \mathrm{m})$ for typical operation of the accelerator at $1.4 \mathrm{MW}$ beam power. In the SNS case, the losses are mainly due to the residual gas in the vacuum chamber. For the FNAL $8 \mathrm{GeV}$ linac and transport line, the stripping losses could be kept below $0.1 \mathrm{~W} / \mathrm{m}$ for $2 \mathrm{MW}$ beam power, provided the $\sim 1 \mathrm{~km}$ long high energy beam transfer line is cooled to $150 \mathrm{~K}$ or lower. Operating the $8 \mathrm{GeV}$ HEBT at room temperature increases the stripping losses to $\sim 1.5 \mathrm{~W} / \mathrm{m}$, mainly due to blackbody radiation; this would exceed the limit loss criterion of $1 \mathrm{~W} / \mathrm{m}$ widely adopted by the accelerator community.

TRACK is a powerful tool for the study of losses in highintensity hydrogen ion linacs, allowing for the simulation of stripping losses, along with losses due to beam mismatch, misalignment, and rf jitter [38]. The stripping mechanisms have been recently implemented in the parallel version of TRACK [39] and high-statistics simulations are now being performed using up to the actual number of particles in the bunch.

\section{ACKNOWLEDGMENTS}

The authors would like to thank C. Hill (FNAL), H. C. Bryant (UNM), and G. H. Herling (UNM) for helpful discussions on the theory of stripping losses from blackbody radiation; T. Williams (SNS) for providing pressure data for the SNS linac and HEBT; T. Anderson (FNAL) for useful discussions on residual gas composition; and $\mathrm{J}$. Galambos and M. Plum (SNS) for providing useful information on SNS beam loss due to stripping. This work is supported by the U.S. Department of Energy under Contracts No. DE-AC02-06CH11357 and No. DE-AC02$07 \mathrm{CH} 11359$.

[1] V.N. Aseev, P.N. Ostroumov, E. S. Lessner, and B. Mustapha, in Proceedings of the 2005 Particle Accelerator Conference, Knoxville, Tennessee, edited by C. Horak (IEEE, Piscataway, New Jersey, 2005), pp. 2053-2055.

[2] N. Catalan-Lasheras, Y. Y. Lee, H. Ludewig, D. Raparia, and J. Wei, in Beam Halo and Scraping: The 7th ICFA Mini-Workshop on High-Brightness Hadron Beams, Lake Como, Wisconsin, 1999, edited by N. V. Mohkov and W. Chou (FNAL, Batavia, Illinois, 2000), pp. 107-111.

[3] T. P. Wangler, E. R. Gray, F. L. Krawczyk, S. S. Kurennoy, G. P. Lawrence, R. D. Ryne, and K. R. Crandall, in Proceedings of the XIX International Linac Conference, Chicago, Illinois, 1998, edited by C.E. Eyberger, R.C.
Pardo, and M. M. White (ANL, Argonne, Illinois, 1998), pp. 657-659.

[4] A. P. Fedotov and B. P. Murin, in Proceedings of the 1976 Proton Linear Accelerator Conference, Chalk River, Canada, 1976, pp. 377-380.

[5] J. Alonso, in Beam Halo and Scraping: The 7th ICFA Mini-Workshop on High-Brightness Hadron Beams, Lake Como, Wisconsin, 1999 (Ref. [2]), pp. 51-61.

[6] J. Honerkamp, Statistical Physics: An Advanced Approach with Applications (Springer, Berlin, 2002).

[7] B. H. Armstrong, Phys. Rev. 131, 1132 (1963).

[8] H.C. Bryant and G.H. Herling, J. Mod. Opt. 53, 45 (2006).

[9] H. C. Bryant (private communication).

[10] H. C. Bryant et al., Phys. Rev. Lett. 58, 2412 (1987).

[11] J. E. Stewart et al., Phys. Rev. A 38, 5628 (1988).

[12] M. L. Du and J. B. Delos, Phys. Rev. A 38, 5609 (1988).

[13] M. L. Du, Eur. Phys. J. D 38, 533 (2006).

[14] J. T. Broad and W.P. Reinhardt, Phys. Rev. A 14, 2159 (1976).

[15] A. R. P. Rau, J. Astrophys. Astron. 17, 113 (1996).

[16] M. A. Furman and D. E. Johnson, Technical Report SSCN-564, SSC/LBL, Berkeley, California, 1988.

[17] L. R. Scherk, Can. J. Phys. 57, 558 (1979).

[18] P. B. Keating et al., Phys. Rev. A 52, 4547 (1995).

[19] W. Chou, Technical Report Beams-doc-2322-v1, Fermilab, Batavia, Illinois, 2006.

[20] N. Solyak and T. Khabiboulline, Technical Report Beamsdoc-3264-v1, Fermilab, Batavia, Illinois, 2008.

[21] J. G. Wang, T. Hunter, D. LeBon, and R. McBrien, in Proceedings of EPAC 2002 Paris: Eighth European Particle Accelerator Conference, edited by T. Garvey, J. Le Duff, P. Le Roux, C. Petit-Jean-Genaz, J. Poole, and L. Rivkin (EPS-IGA/CERN, Geneva, Switzerland, 2002), pp. 2388-2390.

[22] G. H. Gillespie, Phys. Rev. A 15, 563 (1977).

[23] G. H. Gillespie, Phys. Rev. A 16, 943 (1977).

[24] Y. Nakai, T. Shirai, T. Tabata, and R. Ito, At. Data Nucl. Data Tables 37, 69 (1987).

[25] Japan Atomic Energy Agency, Naka Fusion Institute, URL http://www-jt60.naka.jaea.go.jp/english/JEAMDL/code/ 1987.html.

[26] A. Poncet, in CERN Accelerator School: Vacuum Technology, Snekersten, Denmark, 1999: Proceedings, edited by S. Turner (CERN, Geneva, Switzerland, 1999), pp. 165-176.

[27] A. Aleksandrov et al., in Proceedings of Linac 2006: XXIII International Linear Accelerator Conference, Knoxville, Tennessee (ORNL, Oak Ridge, Tennessee, 2006), pp. 145-147.

[28] FermiGrid web site, URL http://fermigrid.fnal.gov.

[29] T. Williams (private communication).

[30] G. W. Foster and J. A. MacLachlan, in Proceedings of EPAC 2002 Paris: Eighth European Particle Accelerator Conference, edited by T. Garvey, J. Le Duff, P. Le Roux, C. Petit-Jean-Genaz, J. Poole, and L. Rivkin (EPS-IGA/ CERN, Geneva, Switzerland, 2002), pp. 826-830.

[31] P. N. Ostroumov, New J. Phys. 8, 281 (2006).

[32] R. L. Madrak and D. Wildman, in Proceedings of Linac 2008: XXIV International Linear Accelerator Conference, Victoria, British Columbia (to be published). 
[33] C. Omet, http://www-aix.gsi.de/ kollmus/Beschl-Pal/ bpalaver_220307.pdf.

[34] R. Kersevan, http://www.vgd07.dl.ac.uk/Presentaions/9th \%20July\%20-\%20morning\%20session/R\% 20Kersevan.pdf.

[35] V. Ziemann, Technical Report SLAC-PUB-5962, Stanford Linear Accelerator Center, Stanford, 1992.

[36] R. Kersevan, Technical Report ST/M-91/17, Sincrotrone Trieste, Trieste, Italy, 1991.
[37] C. Omet and P. Spiller, Technical Report GSI-Acc-Report2004-12-001, GSI, Darmstadt, Germany, 2004.

[38] P. N. Ostroumov, B. Mustapha, and V.N. Aseev, in Proceedings of Linac 2006: XXIII International Linear Accelerator Conference: Knoxville, Tennessee (Ref. [27]), pp. 420-422.

[39] J. Xu, B. Mustapha, V. N. Aseev, and P. N. Ostroumov, Phys. Rev. ST Accel. Beams 10, 014201 (2007). 